\title{
Leopold Figl Observatorium für Astrophysik
}

\author{
Monika Rode-Paunzen \\ Institut für Astronomie, Türkenschanzstraße 17, 1180 Vienna, Austria
}

\begin{abstract}
As soon as the new observatory at the Türkenschanzstraße was build, the scientists noticed the importance for an observing place far away from cities and villages because of dust and light emission. The new observatory in the Vienna wood was built in the $1960^{t h}$ and gives a possibility for astronomers and students to observe without the disturbance from citylight.

This article gives a short overview about the history of creation, buildings and instruments.
\end{abstract}

\section{Baugeschichte}

Kaum war das neue Gebäude der Wiener Universitäts-Sternwarte auf der Türkenschanze seiner Bestimmung übergeben, holten das Streulicht und das Kleinklima der Stadt die Sternwarte ein, wodurch sich die Beobachtungsbedinungen kontinuierlich verschlechterten.

So wurden z.B. Pläne geschmiedet einen größeren Bau als das Wiener Gebäude auf dem Schneeberg, der seit 1897 über eine Zahnradbahn verfügt, zu errichten und die Instrumente zu verlegen. Aber auch die Oberen Tauern oder das Plateau von Ober-Bozen wurden als Standort diskutiert.

Von international renommierten Kollegen, wie z.B. dem amerikanischen Astronomen George Hale, bekamen die Wiener Astronomen Schützenhilfe, doch die ungeklärte Finanzierungsfrage und das mangelnde Interesse von potentiellen Geldgebern ließen alle Pläne in Schubladen versinken.

Trotzdem suchten die Forscher kontinuierlich weiter nach klimatisch begünstigten Beobachtungsplätzen. ${ }^{1}$ So schreibt J. Palisa in "Die Verlegung der

\footnotetext{
${ }^{1}$ Die Zahl der "astronomisch bauchbaren" Nächte liegt in Wien bei maximal $40 \%$. Wegen des hartnäckigen Hochnebels in den kalten Monaten kann man oft vier Monate durchgehend nicht beobachten.
} 
Wiener Sternwarte eine Notwendigkeit" 1924, gegen Ende seiner astronomischen Karriere, daß ein vom Licht ungestörter Beobachtungsplatz für die österreichische Astronomie überlebenswichtig ist.

Der 2.Weltkrieg und die kargen Nachkriegsjahre erlaubten keine Überlegungen zu oder gar die Planung einer astronomischen Außenstation.

In den 1960er-Jahren erhohlte sich die österreichische Wirtschaft und so begann der damalige Sternwartedirektor Josef Meurers, der für seine rege Bautätigkeit bekannt war, 1962 Verhandlungen mit dem Unterrichtsministerium über eine Außenstelle der Sternwarte.

Er konnte den damaligen Unterrichtsminister Theodor Piffl-Perčević von der drigenden Notwedigkeit eines modernen Teleskopes abseits der Großstadt überzeugen und der Minister sagte die Finazierung des Instrumentes durch sein Ministerium zu. In dem niederösterreichischen Landeshauptmann und Altbundeskanzler Leopold $\mathrm{Figl}^{2}$ fand Meurers einen großen Fürsprecher, auf dessen Initiative das Land Niederösterreich die Bereitstellung eines geeigneten Grundstückes und die Übernahme der Kosten für die Errichtung der nötigen Infrastruktur zusagte. Sowohl Teleskop als auch die dazugehörige Infrastruktur wurden 1965 der Universität Wien anläßlich ihres 600-Jahr Jubiläums geschenkt.

\section{Bauplatz}

Nun begann die Suche nach einem geeigneten Bauplatz.

Für Meurers (1966) stand fest, dass der Bau eines nicht ständig besetzten Observatoriums billiger komme, als die Verlegung der gesamten Wiener Sternwarte, und so war die Wien-Nähe des Bauplatzes ein wesentliches Auswahlkriterium. Die Standortbestimmung für ein großes Teleskop ist ein wesentlicher Faktor und bedarf einer genauer Prüfung der lokalen Wetterverhältnisse über einen langen Zeitraum an den in Frage kommenden Aufstellungsplätzen.

Ein Wüstenplateau in großer Höhe läßt sich in Österreich nicht finden. Daß nicht einfach der höchstmögliche Gipfel auch automatisch der beste Beobachtungsplatz ist, zeigte bereits von Rheden 1909 in Folge seiner klimatischen Untersuchungen am Sonnwendstein.

Wichtige Kriterien für den optimalen Standort sind nicht nur eine möglichst hohe Anzahl klarer Nächte, solche geringem Bewölkungsgrad, sondern auch die Reinheit der Luft von Staub etc., die durchschnittliche Luftruhe, Luftfeuchtigkeit und die durch die Nähe großer Siedlungen bedingte Lichtverschmutzung des Himmels. Eine weitere Einschränkung ergab sich, da sich der Bauplatz auf Grund der Schenkung in NÖ befinden mußte.

\footnotetext{
${ }^{2} \mathrm{Ihm}$ zu Ehren erhielt das Observatorium den Namen "Leopold Figl Observatorium für Astrophysik"
} 
Hermann Haupt $(1966,1970)$ widmete sich intensiv der Klimaauswertung von möglichen Standorten in NÖ. In seiner Arbeit (1966) vergleicht er die meteorologischen Aufzeichnungen mehrerer Jahre von sieben potentiellen Bauplätzen mit Höhen zwischen 400-800m und stellt fest, daß das Seeing in mittleren Höhenlagen am günstigsten sind. Paul Jackson (1971) machte die notwendigen Feldmessungen der Luftgüte, Wetterbedingungen und sonstigen Beobachtungsparametern.

Die Wahl fiel auf den $880 \mathrm{~m}$ hohen Mitterschöpfl, der ca. $50 \mathrm{~km}$ von der Universitätssternwarte entfernt im SW von Wien liegt. Da er mitten im geschützten Wienerwald liegt, war und ist ein großer Zuwachs des Siedlungsraumes nicht zu erwarten. ${ }^{3}$ Zudem liegt die durch den Ballungsraum Wien verursachte Himmelsaufhellung im Norden des Beobachtungsplatzes, einer Himmelsrichtung in der eher selten beobachtet wird. Ursprünglich wurde der 893m hohe Schöpfl als Bauplatz angeboten, da sich aber am Kamm der Nebel oft fängt, war und ist er als Standort weniger geeignet.

Das gesamte Gebiet ist zudem Staatsbesitz und so konnte mit den Bundesforsten vereinbart werden, dass alle forstlichen Maßnahmen im Umkreis der Sternwarte nur nach Rücksprache mit den Astronomen durchgeführt würden, um das wichtige Kleinklima rund um das Observatorium nicht ungünstig zu beeinflussen.

Im Herbst 1965 wurde mit dem Bau der Straße begonnen am 13.September 1966 wurde der Grundstein des Hauptgebäudes in Anwesenheit der Witwe Leopold Figls Hilde und vieler Festgäste von Landeshauptmann Eduard Hartmann gelegt. Den Segen sprach wurde Erzbischof-Koadjutor von Wien Dr. Franz Jachym. Mit einem Festakt am 25. September 1969 wurde das Observatorium seiner Bestimmung übergeben. Das "First-Light", die wissenschaftliche Inbetriebnahme, des 1,5m Teleskopes konnte aus organisatorischen Gründen allerdings erst im Dezember 1970 stattfinden.

\section{$1,52 \mathrm{~m}=60 "$ "-Teleskop}

Das Hauptgebäude das den 1,5m Spiegel beherbergt wurde zweckmäßig geplant und gebaut. Es ist ein Einzelturm mit $12 \times 12 \mathrm{~m}$ Grundfläche, $18 \mathrm{~m}$ hoch, mit Keller und 2 Stockwerken, auf den eine doppelwandige Ganzmetallkuppel mit 3m breitem Spalt aufgesetzt ist, die von der VÖST-Linz hergestellt wurde. Der doppelte Kuppelboden bildet eine thermische Barriere gegen den Turm. Zusätzlich kann mittels Ventilatoren, die Außenluft durch den doppelten Boden geblasen und so rasch die Kuppel- an die Außentemperatur angeglichen werden. Die Außenhaut des Gebäudes ist ebenfalls gut isoliert, es gibt keine Fenster an

\footnotetext{
${ }^{3}$ nächtliche Scheinwerfer und Himmelsbeamer, waren damals noch nicht Gegenstand von Überlegungen
} 

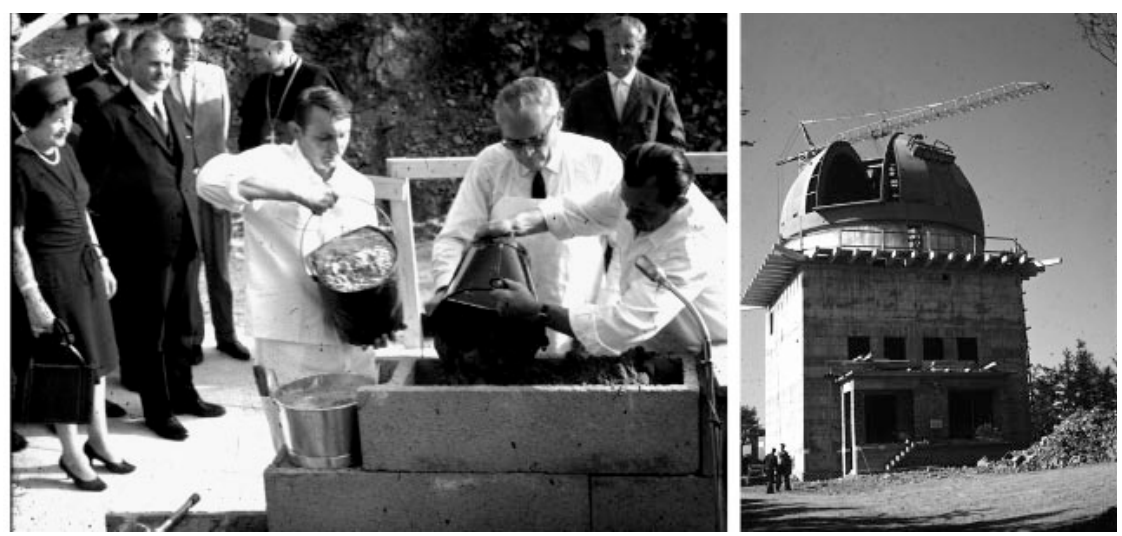

Abb. 1: Links: Grundsteinlegung E.Hartmann, links H.Figl, T.Piffl-Perčević, F.Jachym im Hintergrund / Rechts: Aufsetzen d. Kuppel

der Südseite und die anderen Fenster werden abends mittels Außenrolos lichtdicht geschlossen. So wird thermische Störung und Lichteinfluß gering gehalten. Da geplant war, die Außenstation von Wien aus zu betreiben, Vorbereitungen sowie die Datenreduktion an der Universitätssternwarte durchzuführen, wurde das Pendeln der Beobachter und Mechaniker, bei Bedarf auch täglich, bewußt in Kauf genommen um sich auch so die Personalkosten einer ständigen Besetzung zu ersparen. Deshalb wurden die sozialen Räumlichkeiten spartanisch ausgestattet. Es gibt drei kleine Ruheräume, eine kleine Küche und Sanitäranlage, im ersten Stock, im zweiten Stock und somit unter der Kuppel befindet sich der Coudé-Raum, eine Dunkelkammer, sowie weitere Laborräumlichkeiten, im Erdgeschoß eine Werkstatt und die Aluminisierungsanlage, im Keller befindet sich die Haustechnik und vom Stiegenhaus gut sichtbar der Grundstein.

Das Teleskop (Hooghoudt, 1970) mit offenem Tubus hat eine Gabelmontierung und 2 Sekundärspiegel, die mittels Flip-Flop-Mechanismus leicht gewechselt werden können. Es vereint drei Abbildungssysteme mit je einem RitcheyChrétien-(F/15; Brennweite 22,5m), Cassegrain-(F/8,3; Brennweite 12,5m) und Coudé-Fokus $(\mathrm{F} / 30 ; 45 \mathrm{~m})$, wobei die Coudéanwendung am Fernrohr nie realisiert wurde. Räumlich ist alles vorgesehen. Der Coudéraum, der sich eine Etage unter dem Beobachterfußboden befindet, wird heute als Bibliothek und Arbeitsraum genutzt.

Optik und Fassung wurden von Zeiss in Oberkochen, die mechanischen Teile bei Rademakers-Rotterdam gebaut, Wesemann-Rotterdam erzeugte die Elektronik. 
Es wurde auch eine Aluminisierungsanlage angeschafft um die Ausfallszeiten zu minimieren und langfristig Kosten zu sparen, da die Spiegel in regelmäßigen Abständen eine neue Beschichtung benötigen um ihre Lichtsammelleistung zu erhalten.

Am 26. Oktober 1981 wurde die neue elektronische Steuerung, die von Helmut Jenkner und Manfred Stoll Hard- und Softwaretechnisch entwickelt und gebaut worden ist, in Betrieb genommen. Sie ermöglichte über 25 Jahre eine hervorragende automastische Positionierung und Nachführung des Fernrohres. Zur Zeit wird an einer neuen Steuerung gearbeitet, die die Möglichkeiten der modernen Computertechnik voll ausschöpfen soll.

Mit der immer ausgefeilteren CCD-Technik, die in allen Bereiche der astronomischen Beobachtung Fotografie und Photomultiplier ablöste, wurde es auch für den Schöpfel notwendig ein modernes Endgerät anzuschaffen. Die Wahl fiel auf den Faint Object Spectrograph and Camera, der bereits als DFOSC für Photometrie und Spektroskopie am "Dänischen 1m Teleskop" in Einsatz war. Mit dem Gerät an das eine CCD-Kamera angeshlossen wird, kann während der Nacht zwischen photometrischer und spektroskopischer Beobachtung (und umgekehrt) problemlos gewechselt werden. Die hohe Quantenausbeute der CCD-Kamera verkürzt zudem die Belichtungszeiten und führt dadurch zu einer besseren Ausnutzung guter Beobachtungsnächte.

Der OEFOSC wurde Baugleich zum DFOSC im November 1995 in Kopenhagen fertiggestellt. Am 15. Oktober 1996 wurde er fix am 1,5m Teleskop montiert und ist am 3. November 1996 mit dem First Light in Betrieb gegangen.

Tabelle 1: Teleskope im Vergleich

$\begin{array}{lcc}\text { Durchmesser Hauptspiegel: } & 152 \mathrm{~cm} & 60 \mathrm{~cm} \\ \text { Durchmesser Sekundärspiegel: } & 52 \mathrm{~cm} & 22 \mathrm{~cm} \\ \text { Brennweite: } & 12.5 \mathrm{~m} & 5 \mathrm{~m} \\ \text { Bildfeld (bei angeschl. Instr.): } & 5.75^{\prime} \times 5.75 & 15^{\prime} \times 15 \\ \text { Teleskoptyp: } & \text { Ritchey-Chrétien } & \text { Ritchey-Chrétien } \\ \text { Gewicht: } & 24 \mathrm{t} & 2.2 \mathrm{t}\end{array}$

\section{$60 \mathrm{~cm}$ Teleskop}

Schon bei der Planung des "großen" Spiegels 1969 wurde der Bau eines kleineren Teleskopes auf dem Gelände überlegt.

1973 wurde mit dem Bau des 60cm Teleskopes in der Werkstatt der Wiener Universitätssternwarte begonnen. Insbesondere zeitraubende Gerätetests sollte den großen Spiegel nicht mehr zeitlich blockieren. Daher wurde das kleine 
Fernrohr als kleines Abbild des 60"- Instrumentes, federführend von Ing. Rudolf Pressberger (1979), geplant und ausgeführt. Die Spiegel wurden in der institutseigenen Werkstatt geschliffen, ebenso wurden die Mechanik und Elektronik vor Ort gebaut. Das fertige Teleskop wurde 1979 aufgestellt.

Das 1975 errichtete Gebäude hat eine Grundfläche von 5×5m, der Beobachterfußboden ist $6 \mathrm{~m}$ über dem Gelände eingezogen, die Kuppel aus Aluminium hat einen Durchmesser von 4,5m. Dieses Teleskopgebäude dient nur zum Schutz des Teleskops. Das Teleskop selbst hat einen geschlossenen, quadratischen Tubus, Gabelmontierung und eine elektronisch gesteuerte Nachführung. 1980 erhielt das Fernrohr ein eigens gebautes Photometer. Die photometrische Beobachtung heller Sterne fällt in den Einsatzbereich des Instrumentes. 1981 wurde die extrem langsame Nova PU Vul erstmals mit diesem Instrument beobachtet.

\section{Literatur}

Haupt H., 1970, Wetter und Leben, Jg. 22, S. $89-93$

Haupt H., 1966, Untersuchungen über die klimatische Eignung österreichischer Gebiete für astronomische Beobachtungen, Wetter und Leben 18, 141 Mitt. Univ.-Sternw. Wien 13,S. 37.

Hooghoudt B.G., 1970, Die konstruktive Ausstattung des 60"-Teleskopes, Annalen der Univ.Sternwarte Wien, Bd. 29,2, S. 123

Jackson P., 1971, Die Frage der Ortswahl bei Errichtung einer Außenstation der Universitäts-Sternwarte Wien, Annalen der Universitts-Sternwarte Wien Bd. 30, Nr. 4, S. $65 \mathrm{ff}$

Meurers J., 1970, Symposium anlässlich der Eröffnung des Leopold Figl-Observatoriums, Annalen der Univ.Sternwarte Wien, Bd. 29,2

Meurers J., 1966, Planung und Bau des Leopold-Figl-Observatoriums für Astrophysik der Universitäts-Sternwarte Wien, Sterne und Weltraum Heft 7

Palisa J., 1924, Die Verlegung der Wiener Sternwarte eine Notwendigkeit, Wien, Selbstverlag des Verfassers

Pressberger R., Purgathofer A., Stoll M., 1979, Das neue $60 \mathrm{~cm}$ RC-Teleskop auf dem Mitterschöpfl des Instituts für Astronomie der Universität Wien, Wien, Sternenbote 1979 H. 10, S. $162-165$

Bilder: Privatarchiv Maria.G.Firneis, 